\title{
In-space Data Fusion for More Productive Missions
}

Authors:

Ashish Mahabal, California Institute of Technology, aam@astro.caltech.edu, 6263954201

Trent Hare, Astrogeology, U.S. Geological Survey, thare@usgs.gov

Valerie Fox, University of Minnesota, vfox@umn.edu

Gregg Hallinan, California Institute of Technology, gh@astro.caltech.edu

\section{Endorsers:}

Leslie Deutsch, Jet Propulsion Laboratory, leslie.j.deutsch@jpl.nasa.gov

Richard Doyle, Jet Propulsion Laboratory, richard.j.doyle@jpl.nasa.gov

Josh Vander Hook, Jet Propulsion Laboratory, hook@jpl.nasa.gov

John S Hughes, Jet Propulsion Laboratory, john.s.hughes@jpl.nasa.gov 
Summary: Scientific missions flown today carry limited computational capabilities, intended for minimal data pre-processing and compression, which force data collection to be highly selective and downlink focused. Small data transfer rates from deep space further, and more fundamentally, limit the quantity of data that can be returned to Earth (Deutsch et al., 2016). Increasing computational power onboard spacecraft would significantly increase the quantity of information per bit that can be returned to Earth by enabling data fusion and information summarization beyond simple compression. The lack of modernized radiation hardened hardware has been a major stumbling block for increasing available computational resources. However, technological developments point to changes in the near future to more advanced radiation tolerant chips with higher data capacity (BAE 2017), and require a change in the way that data collection, processing and downlink on spacecraft can be conceptualized. We discuss the merits, capabilities, and applications of enhanced onboard data fusion practices enabled by increased computational resources on scientific missions, in order to empower the community to develop the software infrastructure to capitalize on evolving technological resources.

Nebulae Concept: The Nebulae concept emphasizes the use of a spacecraft not only as a collection of remotely operated instruments, but also as a remote data storage and processing asset - essentially, bringing "the cloud" to outer, even deep space (Vander Hook 2020). This concept also places emphasis on data fusion across instruments and pre-existing data sets to help revolutionize science returns, even before considering the benefits of autonomous operations. Existing and well-vetted techniques and analysis algorithms can be run on these large remote data sets, onboard the spacecraft as confined on the wrong side of the data transfer bottleneck.

For example, the Mars Reconnaissance Orbiter HiRISE camera has imaged and downlinked less than $5 \%$ of the Martian surface, despite covering Mars every few months for the last decade. The Nebulae concept states: leave the camera on and keep your data onboard for immediate and future analysis. Onboard assets then enable the entire data set to be summarized, queried, and mined for information, both as the data coverage is collected and after as it is integrated with other observations. And like a Mars sample-return mission gathering rocks, that same mission could also return the tremendous volumes of collected data back to Earth. But while waiting for that round-trip back to Earth, we can allow the remote system to benefit from that collected data, while also sending back onboard summarized data products and derived analysis results (along with the bandwidth-limited amount of science data as sent today).

What is Data Fusion? Data fusion is the process of synthesizing multiple, potentially very diverse, data sources to allow for more consistent, accurate, and useful information than provided by an individual data source. Data fusion across disparate data sets allows one to distill large volumes of data into information, and thereby improving scientific return and decision making (Figure 1). Data fusion is not just data integration. Attributes from 
multiple instruments are often not uniform, and even for similar instruments data interpolation may often be required. As a result, a higher-level inference or decision process that assesses the reliability of the fused data is required. The principle of data fusion, as applied to space-based data sets, is nothing new. MAPSIT (2020) and Archinal (2016) stress the importance of tying disparate data sets to a well-defined reference frame or coordinate system. The novelty that the community must prepare for is the ability to begin this process onboard the mission, increasing the derived information that can be downlinked even as data transfer rates remain constant.

Implementing Broadly Applicable Fusion Capabilities: The ability to successfully fuse data is highly dependent on having clear and common expectations of the contents, context and meaning of that data. With onboard assets using data from multiple data sources, data "interoperability" subsequently becomes a significant problem within the data fusion process (Hare 2017).

Research shows that ontologies can be used to formally represent knowledge within domains and enable better interoperability by allowing data to be linked at the structural, syntactic and semantic level. These levels respectively address the different ways of storing data, formatting the data, and interpreting the meaning of the data. In particular the meaning of the data, 'Semantic interoperability', has been defined as ensuring that the precise meaning of exchanged information is understandable by any other system or application not initially developed for this purpose (Commission 2008).

Ontologies have been used to successfully develop the archival standards for the Planetary Data System (PDS, Hughes 2014), NASA's official archive for Solar System Exploration data. The PDS ontologies define the concepts required for long-term preservation and reuse of the science data in accordance with principles from the Open Archive Information Systems (OAIS) Reference Model (ISO 14721:2012). These ontologies define concepts in topics covering cartography, flight geometry, and data formats, and should be extended to enable in-space data fusion.

\section{Applying the Nebulae Concept}

Cross Instrument Fusion: Bringing additional computational power earmarked for data processing and pre-downlink analysis fundamentally changes the way that space exploration missions can be formulated by enabling cross-instrument integration. Every mission would be able to tailor, depending on the instruments it has, a set of top science-oriented queries for combining the diverse data being generated (Gray et al 2002), and leverage onboard assets to optimize downlink of summarized products. This synthetic model allows for more dynamic response to discovery, and enables observation modes that are difficult to capture with a traditional pre-targeted, selective, operational mode. Flexible computational power can also better allow spacecraft to adapt operations to enable cooperation between multiple missions (e.g. a rover and an orbiter), distributing processes 
to be more efficient. As the technology evolves, distributed processing in the form of a dedicated server spacecraft for multiple assets could finalize the transition of the cloud to outer space.



Figure 1. A simple data fusion model including topography, roughness, and thermal inertia to ascertain landing site suitability based on weighted results for each constraint. Topography might be an "absolute" constraint to determine a suitable elevation, and thermal inertia could be weighted from "optimal" to "questionable" to "unacceptable" as a proxy for surface rock and dust cover. The last step in the model further constrains the landing areas (called landability) to areas actually safe enough to send the spacecraft based on enough contiguous space to accommodate the defined landing ellipse.

Leveraging Existing Analysis: Taking advantage of higher onboard data capacities will allow for loading existing and calibrated data, prior to launch, to facilitate onboard corrections for the newly acquired data (e.g. updated registration). Capabilities for onboard data fusion of existing and freshly taken data will open up many possibilities for new science. For example, time series and longitudinal data can help quickly discover dynamic features which would likely be missed without immediate onboard processing or the required delay to transfer and process the data back to Earth.

For worlds that have been visited only once or twice, limited amounts of existing data will be available; thus, packing them on board will not be too difficult. But for worlds that have been visited a large number of times like Mars or the Moon, loading all past data onboard the spacecraft will just not be reasonable. The data can be organized to be 
accessible on demand in a summarized format. A querying system can be used to transport limited amounts of data back and forth between Earth and the distant spacecraft for combining recent data with archival data from Earth received in response to relevant queries. This can be simplified by building standardized ontologies and structures built over the metadata (e.g. Mahabal et al. 2001).

\section{Scientific Applications:}

Planetary Science Examples: High resolution imaging and topographic information are fundamental data sets acquired when exploring new celestial bodies, but developing global coverage and accurate models require significant downlink. In this current schema, stereo images are gathered on Mars and returned to Earth. Complete processing requires sensor calibration, updated spatial position as controlled to an existing base map, and the derivation of an elevation surface to realize the ability to topographically correct the original input images such that they are tightly aligned to a known location (Figure 2). With onboard processing and the ability to co-align the data to an existing data set, a much larger amount of data could be automatically processed onboard to facilitate surface monitoring or change detection as global data sets are developed (e.g. weather monitor for active rovers, locating new impact craters, or tracking recurring slope lineae (RSL) and polar C02-driven geomorphological processes). Even though Mars is relatively nearby, we continue to find ourselves limited by the communications link to Earth. Moving more of the information consolidation to the other side of the bottleneck can result in substantial increases in our understanding of global and temporal phenomena. Mars will continue to be a target for exploration, and therefore will generate orders of magnitude more science data and information from existing and future missions.

As missions move farther from Earth, automated workflows become far more critical. Missions to the Jovian system and beyond contend with round-trip communication time in multiple tens of minutes and trickling data transfer rates. On dynamic worlds like Io, carrying existing maps and the ability to calibrate onboard new acquired data provides an opportunity to rapidly compute rates of change. Being able to track one day or even one month's worth of change on Io could be as dramatic as current mapping efforts of Southern California (or other parts of Earth) showing the surface actually rising and falling as a function of season and aquifer changes (Parker 2016).

NASA's latest New Frontiers mission, Dragonfly, will be sending an octocopter to explore the surface of Titan, and demonstrates the utility of onboard data processing. The mission will have LiDAR, radar, cameras and many more mobility support instruments. However, due to bandwidth, processing, and storage limitations, only a subset of the sensor data collected in flight will be retained onboard or even transmitted back to Earth. If a Nebulae-like system were available, it would enable the constant collection of sensor data for storage and analysis, maintaining all the potential critical data collected during the 
mission. Onboard data fusion techniques could help more safely guide the octocopter and can greatly increase science return.

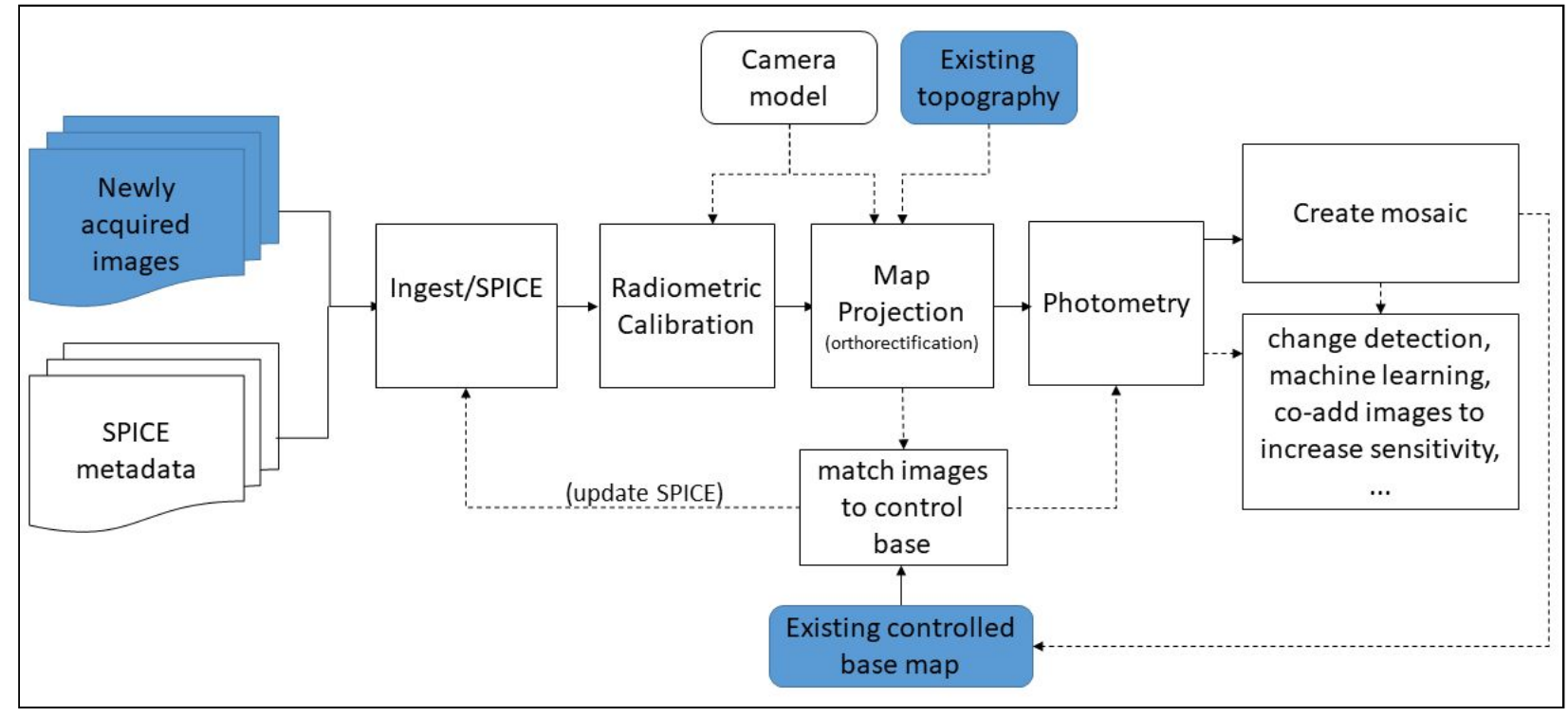

Figure 2. A photogrammetric workflow, for example as used for surface images on Mars, which utilizes preloaded data sets (and a feedback loop for the data itself) to facilitate processing of newly acquired images. This pipeline requires data fusion capabilities to help calibrate and spatially align the newly acquired data such that they can be immediately used for onboard analysis (e.g. change detection). Image updated from Vander Hook 2020.

Astronomy Cases: The volume of astronomy data captured by missions has been rising exponentially. We still rely on processing on Earth for data collected from diverse missions. The processing often includes combining the latest data with older data, or data from other instruments/missions. As an example, the Transiting Exoplanet Survey Satellite (TESS) has large pixels ( 21"x21") and the primary contaminants in its search for Planet Candidates (PC) are Eclipsing Binaries (EB) which can often be in the same pixel as the target PC. The data collected are transferred to Earth once a month when the satellite is closest to Earth in its oblong orbit. If the satellite hosted an all-sky catalog with good astrometric precision (or two catalogs, with the inclusion of a color term), reducing potential contaminants at source with a small bit of computation would be trivial. In fact, to reduce the required data holding, just the high-precision catalogs of the next sector can be uploaded every time the satellite passes close to Earth. Better still, the orbit could have been designed differently and the requirement for close fly-bys and resulting gaps in data avoided by carrying a dataset, cross-matching it with fresh data, and reducing the download requirements to smaller volumes of summarized and detrended data through onboard processing. Another unfortunate incident which might have been resolved involved the forced dumping of quasar data, as taken by Kepler, due to lack of downlink bandwidth back to Earth. Availability of recent variability of quasars, or long-term variability catalogs would have ensured that at least the data for most active quasars would have been achieved. 
The availability of in-situ computing resources will continue to limit the potential scientific scope of future missions. By way of example, we highlight the Probe-class concept FARSIDE (Burns et al. 2019), a proposed low radio frequency interferometric array on the farside of the Moon. This notional architecture consists of 128 dipole antennas deployed across a $10 \mathrm{~km}$ area by a rover, and tethered to a base station for central processing, power and data transmission to the Lunar Gateway, or an alternative relay satellite. The aggregate data rate from all antennas to the base station amounts to $1.7 \mathrm{~PB} /$ day. The data are averaged down to a time resolution of 60 seconds for a manageable data rate for transmission of $65 \mathrm{~GB} /$ day. These data are then used to image the entire viewable sky at this cadence from the lunar farside, enabling near-continuous monitoring of the nearest stellar systems in the search for the radio signatures of coronal mass ejections and energetic particle events, and the magnetospheres for the nearest candidate habitable exoplanets. However, these phenomena are expected to exhibit variability on shorter timescales, and enhanced science is immediately possible with i) a faster downlink, or ii) the presence of a ring buffer on the lunar FARSIDE that stores high time resolution data products sufficiently long for event detection at Earth to occur. After detection, higher time resolution data is pulled from the ring buffer for transmission to Earth. With more compute capabilities and the possibility of data fusion on the lunar surface, better data products can be downlinked, leading to enhanced science.

\section{Recommendations:}

Recommendation 1: Design future instruments bearing in mind that local processing, greater storage volumes, and fusion capabilities will be available onboard. Employing onboard processing allows for more data collection than could ever potentially be returned to Earth in raw form. These data can be summarized and analyzed onboard to help prioritize their science utility, consolidate information, and store for downlink or eventual full transfer via a "data-return" mission.

Recommendation 2: Design mission science goals keeping data fusion capabilities in mind. Reverse the paradigm that designs scientific missions to meet data downlink constraints into a paradigm that collects the data desired by scientific pursuits. Broaden future missions to allow for derived product creation and autonomous capabilities, as made possible by high data collection rates and onboard processing.

Recommendation 3: Equip missions with calibrated and accurately located summary datasets, information, and analysis algorithms onboard. This pre-loaded inventory can then be used to help calibrate, spatially align, and further enhance newly acquired data sets onboard the spacecraft by using the same processing workflows defined and already in use by instrument teams on the ground.

Recommendation 4: Redesign data fusion code and libraries currently available on Earth to work more autonomously. For example, many processing pipelines enable automatic 
image-to-image matching to align newly acquired images to an existing base map. Continue to research and enhance those existing techniques capabilities to be more robust and dependable within autonomous systems, with the intention of applying these workflows to space-based platforms.

Recommendation 5: Standardize ontologies to provide a framework for diverse data fusion, and identify common queries and codify them for fast implementation for summarized data exchange between Earth and remote missions. Simple summary products, be it low-resolution images, an identified change detection, or statistical reports can quickly help determine when critical data products can be prioritized for downlink.

\section{References:}

Archinal, B. A., et al. (2016), Registering Planetary Datasets for Data Fusion: A "Force Multiplier" for Planetary Science, 47th Lunar and Planetary Science Conference, URL: https://www.hou.usra.edu/meetings/lpsc2016/pdf/2377.pdf

BAE (2017) Radiation-hardened electronics product guide, URL: https://www.baesystems.com/en-us/download-en-us/20200326131922/1434554723601.pdf

Commission Recommendation of 2 July 2008 on cross-border interoperability of electronic health record systems (notified under document number C(2008) 3282).

Deutsch, L., Townes, S., Liebrecht, P., Vrotsos, P., and Cornwell, D., (2016) Deep Space Network: The Next 50 Years, SpaceOps Conference, Marseille, France.

Gray et al., (2016) Data Mining the SDSS SkyServer Database, URL: https://www.microsoft.com/ en-us/research/wp-content/uploads/2016/02/tr-2002-01.pdf

Hare, T.M.; Rossi, A.P.; Frigeri, A.; Marmo, C. (2017) Interoperability in Planetary Research for Geospatial Data Analysis. Planet. Space Sci. doi:10.1016/j.pss.2017.04.004

Hughes, J.S., D Crichton, S. Hardman, E. Law, R. Joyner, P Ramirez, "PDS4: A model-driven planetary science data architecture for long-term preservation," Data Engineering Workshops (ICDEW), 2014 IEEE 30th International Conference on Data Engineering Workshops, pp.134,141, March 31 2014-April 42014.

Mahabal, A, Djorgovski,S.G., Brunner, R.J., Williams, R.E., "Topic maps as a virtual observatory tool," Proc. SPIE 4477, Astronomical Data Analysis, (1 November 2001); https://doi.org/10.1117/12.447170 URL: https://arxiv.org/pdf/astro-ph/0110184.pdf

Mapping and Planetary Spatial Infrastructure Team (2020), URL: https://www.lpi.usra.edu/mapsit/

Burns, J.O., et al. (2019) NASA Probe Study Report: Farside Array for Radio Science Investigations of the Dark ages and Exoplanets (FARSIDE), Nov. 2019, https://arxiv.org/abs/1911.08649

Parker, A. L. , Biggs, J. , \& Lu, Z. (2016). Time-scale and mechanism of subsidence at Lassen Volcanic Center, CA, from InSAR. Journal of Volcanology and Geothermal Research, 320, 117-127. 10.1016/j.jvolgeores.2016.04.013

Vander Hook, Joshua and Castillo-Rogez, Julie and Doyle, Richard et al. (2020) Nebulae: A Proposed Concept of Operation for Deep Space Computing Clouds. In: 2020 IEEE Aerospace Conference. IEEE , Piscataway, NJ, pp. 1-14. ISBN 978-1-7281-2734-7. URL: https://resolver.caltech.edu/CaltechAUTHORS:20200908-130820600 